\title{
Contactless exploration of graphene properties using millimeter wave response of WGM resonator
}

\author{
A. A. Barannik, N. T. Cherpak, I. A. Protsenko, A. I. Gubin, D. Kireev, and S. Vitusevich
}

Citation: Appl. Phys. Lett. 113, 094102 (2018); doi: 10.1063/1.5041335

View online: https://doi.org/10.1063/1.5041335

View Table of Contents: http://aip.scitation.org/toc/apl/113/9

Published by the American Institute of Physics

\section{Articles you may be interested in}

Resonant metasurface with tunable asymmetric reflection

Applied Physics Letters 113, 094103 (2018); 10.1063/1.5046948

Frequency-dependent transmission-type digital coding metasurface controlled by light intensity

Applied Physics Letters 113, 091601 (2018); 10.1063/1.5045718

Functional gradient effects on the energy absorption of spider orb webs

Applied Physics Letters 113, 103701 (2018); 10.1063/1.5039710

Droplet ski-jumping on an inclined macro-textured superhydrophobic surface

Applied Physics Letters 113, 103702 (2018); 10.1063/1.5048301

Diamond photovoltaic radiation sensor using pn junction

Applied Physics Letters 113, 093504 (2018); 10.1063/1.5034413

Dual-resonator Lamb wave strain sensor with temperature compensation and enhanced sensitivity Applied Physics Letters 113, 093502 (2018); 10.1063/1.5046120

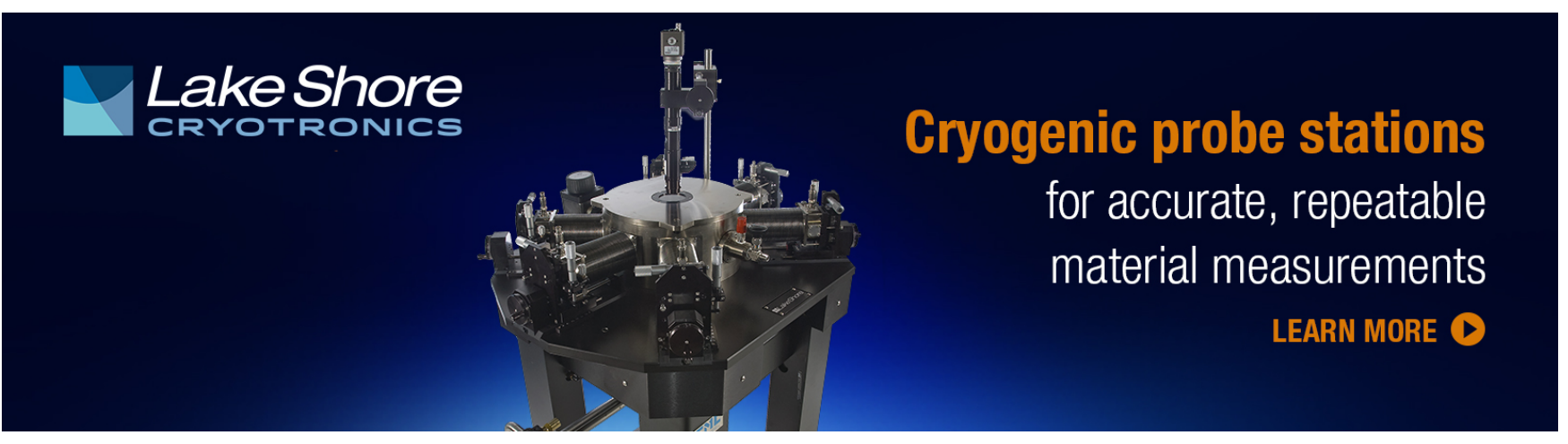




\title{
Contactless exploration of graphene properties using millimeter wave response of WGM resonator
}

\author{
A. A. Barannik, ${ }^{1,2}$ N. T. Cherpak, ${ }^{1}$ I. A. Protsenko, ${ }^{1}$ A. I. Gubin, ${ }^{1,2}$ D. Kireev, ${ }^{2}$ \\ and S. Vitusevich ${ }^{2, a)}$ \\ ${ }^{1}$ O.Ya. Usikov Institute for Radiophysics and Electronics, NAS of Ukraine, 61085 Kharkiv, Ukraine \\ ${ }^{2}$ Institute of Bioelectronics (ICS-8), Forschungszentrum Juelich, 52425 Juelich, Germany
}

(Received 24 May 2018; accepted 13 August 2018; published online 29 August 2018)

\begin{abstract}
The response of a sapphire whispering gallery mode (WGM) resonator to a single-layer graphene film was studied in the millimeter wave band (frequency of about $40 \mathrm{GHz}$ ) at different distances of graphene from the resonator. In the resonator, the $\mathrm{HE}_{141 \delta}$ WGM was excited, in which the longitudinal component of the electric field is predominant. Based on the fitting results of both the response measurement and the numerical simulation of the resonator, the conductivity value was obtained for a known film thickness. The conductivity of our CVD-grown and transferred graphene was found to be $(1.02 \pm 0.06) \times 10^{6} \mathrm{~S} / \mathrm{m}$. This deviates slightly from the values obtained through our DC conductivity measurements, reflecting the real parameters of the graphene material after transfer from copper to a quartz substrate. A significant difference was demonstrated between the conductivity values obtained by the fitting procedure and those calculated using the perturbation method. In explanation for the discrepancy, we propose a possible inapplicability of the perturbation method for the cases of both the resonator and mode polarization used in this work. The results of this work show that a WGM resonator technique allows contactless exploration of graphene parameters, such as conductivity or sheet resistance, in the millimeter wave band. Published by AIP Publishing.

https://doi.org/10.1063/1.5041335
\end{abstract}

Graphene has excited a huge wave of research activity in physics and materials science. Much work has been done for studying the physical properties of graphene and many suggestions have been made for potential applications, ${ }^{1}$ in particular, in nanotechnology and bioelectronics (see, e.g., Refs. 2 and 3). The appearance of large-area graphene films has attracted great interest among researchers, including those who studied the microwave properties of ultrathin conductive films (see, e.g., Ref. 4).

The first results of the microwave response of a resonator with graphene films were reported in $2010 .^{5,6}$ They showed that the surface conductivity of the sample $\sigma d_{f}$ is convenient for analyzing the response, where $\sigma$ is the specific conductivity and $d_{f}$ is the thickness of the film. Furthermore, "dielectric" behavior of the graphene film occurs for values $\sigma d_{f}<10^{-2}$ S. ${ }^{5}$ Previous work also underlined the importance of the azimuthal component of the electric field in the region where graphene is located. Moreover, the technique allows a robust and contactless evaluation of large-area graphene conductivity $\sigma$ using dielectric resonators (DRs) with the $\mathrm{TE}_{01 \delta}$ mode providing information on the film thickness $d_{f}$.

The problem of the contactless method for measuring the graphene conductivity $\sigma$ using DR with the same mode was formerly considered by Hao et al. and Shaforost et al..$^{7-9}$ The aforementioned authors prove the advantage of their technique for finding the electrical parameters of a nm-thick film without the electrodynamic model of finite elements. The field distribution in the resonator structure was calculated using COMSOL finite-element software. ${ }^{8}$ It should be

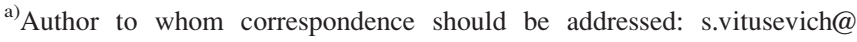
fz-juelich.de
}

noted that all of the aforementioned studies ${ }^{5-9}$ were performed in the $\mathrm{TE}_{01 \delta}$ mode with a frequency band below $15 \mathrm{GHz}$, where the dynamic and DC electronic conductivity values are equal with high accuracy.

However, a higher frequency range including millimeter $(\mathrm{mm})$ waves has not yet been explored for graphene materials. When studying the microwave properties of thin films of metal and graphene materials, the important parameter is the Q-factor of the resonator. In this regard, it is especially important to investigate the effect of introducing graphene into the structure of a high-Q DR in which the whispering gallery modes (WGMs) are excited. It should be emphasized that in a Ka-band, a sapphire WGM resonator has relatively high intrinsic Q-factor of up to $4 \times 10^{4}$ (frequency $\mathrm{f} \cong 40 \mathrm{GHz}$ and $\mathrm{T}=300 \mathrm{~K}) .{ }^{10}$ This manuscript was preceded by other publications $^{11,12}$ on the investigation of a WGM sapphire resonator with ultrathin copper films, demonstrating peculiarities at small copper thicknesses.

In this work, we report the experimental results of studying the response characteristics in the Ka-band WGM sapphire resonator to single-layer graphene at different distances of graphene from the resonator. These results are then compared with results obtained by numerical simulations. The numerical modeling of a resonator with and without ultrathin graphene films was performed using the radiofrequency module of COMSOL Multiphysics. ${ }^{13}$ The open boundary was realized by combining the scattering boundary condition and the perfectly matched layer. Graphene films were specified using the transition boundary condition (TBC), which is recommended by the developers for modeling thin metallic materials. The description of the approach to simulation using the "transition boundary condition" can be found in Ref. 13. 
TBC is appropriate to model films with thicknesses of about skin depth. The model allows the eigenvalues of complex frequency to be obtained when varying the distance of graphene from the resonator, the electrical conductivity with known graphene thickness, and it allows us to assess the quality of graphene in a contactless manner.

The graphene used in this work was purchased from Graphenea. ${ }^{14}$ Graphene was CVD-grown on an $18 \mu \mathrm{m}$ thick copper foil. Raman spectroscopy, as provided by the company, revealed a predominant $(>98 \%)$ single-layer coverage $\left(\mathrm{I}_{\mathrm{G}} / \mathrm{I}_{2 \mathrm{D}}<0.5 ; \mathrm{I}_{\mathrm{D}} / \mathrm{I}_{\mathrm{G}}<0.05\right)$ with sparse bilayer patches. In order to transfer graphene onto arbitrary substrates, e.g., sapphire or quartz, a layer of PMMA $(300-500 \mathrm{~nm})$ was first spin-coated on top of the graphene-on-copper in order to serve as a support polymer and oxygen plasma $(0.8 \mathrm{mbar}$, $100 \mathrm{~W}, 5 \mathrm{~min}$ ) was used to remove graphene from the backside of the foil. After this, the PMMA/graphene/Cu stack was moved to a copper etchant solution $\left[0.1 \mathrm{M}\left(\mathrm{NH}_{4}\right)_{2} \mathrm{~S}_{2} \mathrm{O}_{8}\right]$ for $24 \mathrm{~h}$ and subsequently subjected to several deionized (DI) water washing steps. When the PMMA/graphene stack was fished out by the target substrate, it was left for $24 \mathrm{~h}$ under ambient conditions to slowly dry out followed by annealing at $160^{\circ} \mathrm{C}$ for $10 \mathrm{~min} .{ }^{15}$ When the structure had cooled, PMMA was dissolved in acetone $\left(1 \mathrm{~h}\right.$ in $50^{\circ} \mathrm{C}$ acetone followed by $12 \mathrm{~h}$ in cold acetone). Finally, the structure was washed with isopropanol and DI water, dried under nitrogen flow, and annealed at $350^{\circ} \mathrm{C}$ in a $\mathrm{N}_{2}$ atmosphere.

The WGM resonator is a sapphire $\left(\mathrm{Al}_{2} \mathrm{O}_{3}\right)$ cylindrical disk with sizes: $2.5 \mathrm{~mm}$ height and $14.25 \mathrm{~mm}$ diameter, excited by dielectric waveguides [see Fig. 1(a) and Ref. 10 for experimental setup details as well as advantages of WGM resonator technique]. The optical axis of the sapphire is parallel to the geometric axis of the disk. Using the corresponding orientation of the waveguides with respect to the cylindrical surface of the disk allowed us to excite the $\mathrm{HE}_{\mathrm{nm} \delta} \delta$ mode, in which the dominant electric field is aligned along the cylinder axis. Here, $\mathrm{n}, \mathrm{m}$, and $\delta$ are the azimuthal, radial, and axial indices, respectively. For this mode, the longitudinal component of the electric field exceeds the azimuthal, at least inside the dielectric disk. ${ }^{10}$ For the microwave measurements, a vector network analyzer N5230A was used. The response was measured for the following cases: (i) a bare sapphire-based disk resonator without a substrate [Fig. 1(b)], (ii) with a $1 \mathrm{~mm}$ thick quartz substrate without graphene [Fig. 1(c)], and (iii) with a graphene film deposited on a quartz substrate [Figs. 1(d) and 1(e)]. The graphene film is placed on a quartz
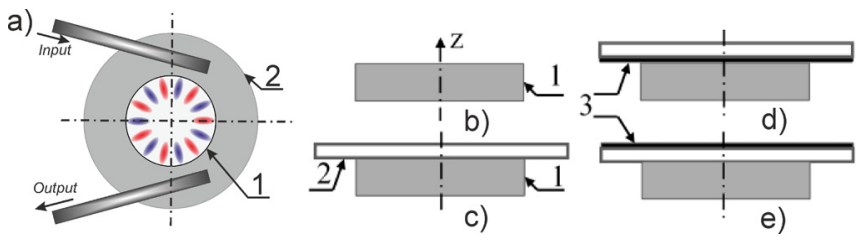

FIG. 1. (a) WGM resonator, excited by dielectric waveguides: input and output, the resonator structures consisting of a sapphire disk 1 (the ellipses represent the $\mathrm{HE}_{141 \delta}$ mode), a bare quartz substrate 2 and a graphene film 3 . Here, (b) and (c) represent the schematics of just the sapphire disk and sapphire with a bare quartz substrate, respectively. (d) and (e) Depict situations where graphene is located at the bottom and at the top of the quartz substrate, respectively. substrate of $30 \mathrm{~mm}$ diameter. In order to study the effect of the topological location of graphene on the WGM resonator, the quartz/graphene substrate was used in two configurations: (i) the graphene film is located directly on a plane of the disk [graphene position "bottom," Fig. 1(d)] and (ii) the graphene film is separated from the disk by a substrate [graphene position "top," Fig. 1(e)].

The measured response in the form of $\mathrm{S}_{21}$ transmission versus the frequency for $\mathrm{HE}_{141 \delta}$ mode is shown in Fig. 2, where the resonance lines were analyzed using a special computer program developed in IRE NAS, Ukraine. With $\mathrm{S}_{21}$ being the signal transmitted between two waveguides during the frequency scan up to $40 \mathrm{GHz}$, it is possible to distinguish and study the resonances, their Q-factors and frequency position. The plots of measured $\mathrm{S}_{21}$ for the $\mathrm{HE}_{141 \delta}$ mode resonator in bare, "graphene on top" and "graphene on bottom" configurations are shown in Fig. 2. There is an obvious shift of resonance frequencies upon addition of a single layer of graphene to the system.

As can be seen from Fig. 2, the resonance curve becomes broader and less intense as the film approaches the flat surface of the sapphire disk (compare curves for "top" and "bottom" positions). This reflects the fact that bringing graphene closer to the resonator's surface (position "bottom") significantly enhances power dissipation within graphene. More complete data on the measured microwave response for both positions of the graphene film are given in Table I.

In Table I: $Q_{0}, Q_{S}$, and $Q_{G}$, and also $f_{0}, f_{S}$, and $f_{G}$ are the Q-factors and frequencies of the resonator corresponding to the following cases: bare resonator, resonator with a substrate, and resonator with graphene on the substrate, respectively. The parameters in Table I allow us to find the $\sigma$ of graphene material of known thickness. To do this, the measured values of the aforementioned Q-factors and COMSOL simulation data were compared. It is known that the graphene thickness is equal to $0.4 \mathrm{~nm} .^{5-9}$ The fitting procedure in the case of both positions gives the conductivity $\sigma=(1.02 \pm 0.06) \times 10^{6} \mathrm{~S} / \mathrm{m}$.

To model a resonator structure containing a thin conductive film on a substrate, two parameters, film's conductivity

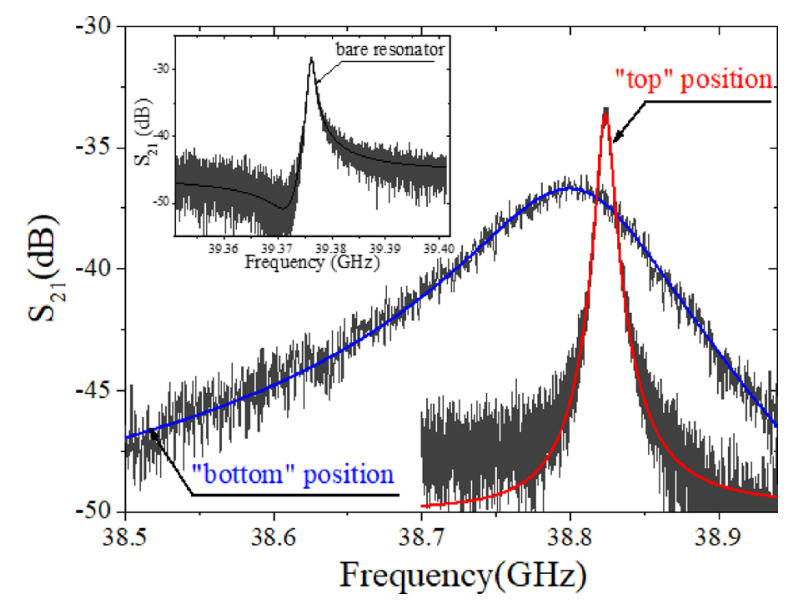

FIG. 2. Measured $\mathrm{S}_{21}$ coefficient dependence on the frequency for $\mathrm{HE}_{141 \delta}$ mode. Broad and narrow resonance lines correspond to the positions "bottom" and "top" [see Figs. 1(d) and 1(e)] of graphene, respectively. The $\mathrm{S}_{21}$ transmission for the bare resonator [see Fig. 1(b)] is shown in the inset. Solid lines (blue and red) show the results of special computer processing of the measured $S_{21}$ coefficient. 
TABLE I. Summary of microwave response parameters of the sapphire WGM resonator with the quartz substrate and the single-layer graphene material.

\begin{tabular}{lcccccc}
\hline \hline$Q_{0}$ & $f_{0}(\mathrm{MHz})$ & $Q_{S}$ & $f_{S}(\mathrm{MHz})$ & Position & $Q_{G}$ & $f_{G}(\mathrm{MHz})$ \\
\hline 33676 & 39376 & 25387 & 38808 & Top & 3778 & 38824 \\
& & & & Bottom & 316 & 38809 \\
\hline \hline
\end{tabular}

$\sigma$ and film's thickness $d_{f}$, were taken into account. Figure 3 shows an example of such modeling of the resonator when a conducting film's thickness is varied in the range from $10^{-4} \mathrm{~nm}$ to $10^{4} \mathrm{~nm}$ in a top position [see Fig. 1(d)]. The two conductivity values, $1.02 \times 10^{6} \mathrm{~S} / \mathrm{m}$ and $1.02 \times 10^{7} \mathrm{~S} / \mathrm{m}$, are typical for graphene and they were taken to compare the results of modeling for thin-film materials of different conductivities to resemble graphene behavior.

It is obvious that the Q-factor plots have a certain minimum level at specific thicknesses that depend on the film's $\sigma$. Increasing $\sigma$ shifts the plot to the left. Consequently, one can conclude that increased conductivity increases losses in the resonating system. It should be noted that substantial changes/shifts in the resonance frequency also occur in the region of the Q-factor's minima, which, according to previous works can be explained by the "dielectric" behavior of the ultrathin conductive films. ${ }^{5,9,12}$

We fit our experimental data (Fig. 2 and data shown as open circles and filled triangles in Fig. 3) with the COMSOL model for graphene as an ultrathin conductor with $d_{f}=0.4 \mathrm{~nm}$. The fitting results allow the precise estimation of graphene conductivity value. In our case, we obtained $\sigma=1.02 \times 10^{6} \mathrm{~S} /$ $\mathrm{m}$. This value slightly deviates from the values obtained through DC conductivity measurements reflecting the real parameters of graphene material after transferring from copper to quartz substrate.

Additionally, there may be a question concerning the applicability of COMSOL-based modeling for the simulation of ultrathin conductive films, especially those with a structure that is just one atom thick. To unravel this issue, we

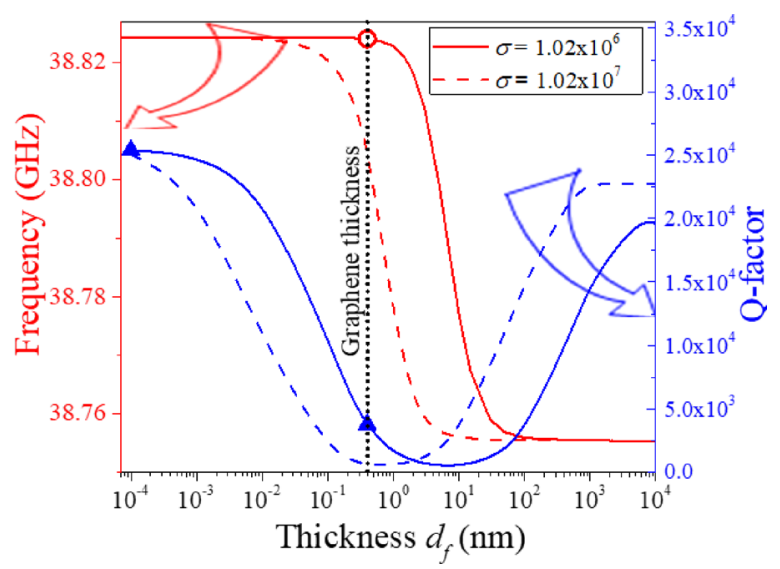

FIG. 3. Measured resonator frequency $f_{g}(\bigcirc)$ and Q-factor $Q_{g}(\boldsymbol{\Delta})$ of DR for "graphene on top" configuration. Solid and dashed lines represent the numerical modelling of the resonance frequency and Q-factor dependence on the thickness of a film for film conductivity values $\sigma=1.02 \times 10^{6} \mathrm{~S} / \mathrm{m}$ and $\sigma=1.02 \times 10^{7} \mathrm{~S} / \mathrm{m}$, respectively. The solid line $\left(\sigma=1.02 \times 10^{6} \mathrm{~S} / \mathrm{m}\right)$ fits the experimental points. The utmost point on the left corresponds to the resonator Q-factor with a substrate without graphene. simulated the resonator for a set of distances between the substrate with graphene and the resonator and compared the results with the experimental data. Figure 4 shows the main resonator parameters as functions of the distance between the sapphire disk flat base and the quartz substrate with graphene on top. There is indeed very good agreement between the measured and calculated data.

The sheet resistance can be estimated using the following formula $R_{s h}=1 /\left(\sigma d_{f}\right)$. Assuming a graphene thickness of $0.4 \mathrm{~nm}$, we find $R_{s h}$ to be equal to about $2.45 \mathrm{k} \Omega / \square$. The value of $R_{s h}$ estimated using our technique matches with the results of other work, where estimated $R_{s h}$ at frequency Xband spans from $26 \Omega / \square$ for SiC-based graphene to $13 \mathrm{k} \Omega / \square$ for CVD-grown graphene and up to $48 \mathrm{k} \Omega / \square$ for reduced graphene oxide. ${ }^{7}$ On the other hand, the $R_{s h}$ values provided by the supplier are in the range of $300-800 \Omega / \square$ as achieved through van der Pauw measurements. ${ }^{14}$ Such a discrepancy between $R_{s h}$ values can be explained by many factors, such as the frequency of electromagnetic fields, differences in transfer techniques, possible incomplete removal of PMMA, partial doping of graphene or even mechanical deformations during the transfer. ${ }^{15}$ Nonetheless, the sheet resistance estimated through our numerical model reflects the actual properties of large scale CVD grown graphene.

In order to further consolidate our experimental data, we applied another approach proposed by Hao et al. ${ }^{7}$ The model, however, is designed for $\mathrm{TE}_{011}$ mode resonators that have only an azimuthal component and it allows the calculation of $R_{s h}$ without the need to identifying the electromagnetic mode in the DR. Under reasonable assumptions, the relation can be presented in a modified form as follows:

$$
\sigma=\frac{2 \pi f_{o}^{2} \varepsilon_{o}\left(\varepsilon_{s}^{\prime}-1\right)}{\Delta f_{s}} \frac{t_{s}}{d_{f}}\left(\frac{1}{Q_{g}}-\frac{1}{Q_{s}}\right)
$$

where $\varepsilon_{s}^{\prime}$ is the real part of the complex permittivity of the substrate $\Delta f_{s}=f_{o}-f_{s}$. The value of conductivity calculated by Eq. (1) is $2.4 \times 10^{5} \mathrm{~S} / \mathrm{m}$, which is almost four times lower than the value estimated by our COMSOL-based modeling

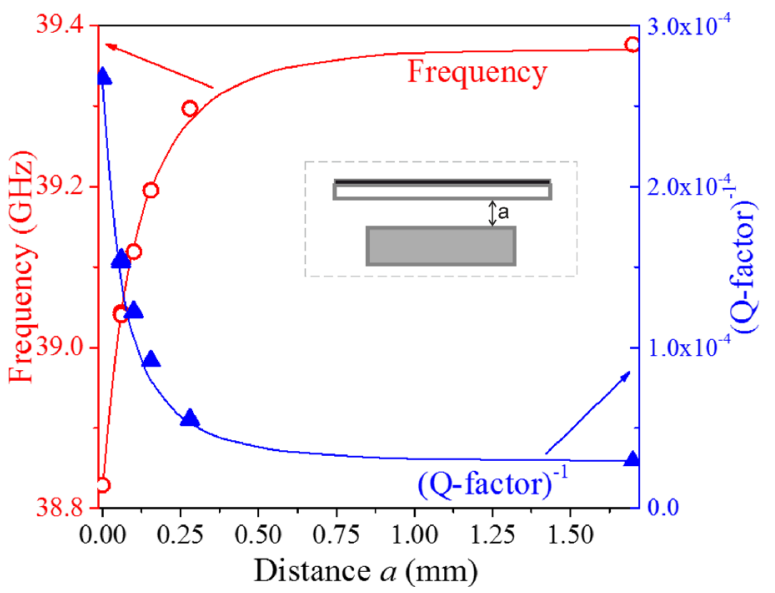

FIG. 4. Resonator frequency $f_{g}$ (red circle) and inverse Q-factor $Q_{g}$ (blue triangle) with graphene in the "top" position as a function of independence of the distance $a$ (see inset into the figure) between a plane of the sapphire disk and a substrate, $\sigma=1.02 \times 10^{6} \mathrm{~S} / \mathrm{m}$. The open circles and closed triangles show the results obtained experimentally, while the lines show the results of the calculations. 
$\left(1.02 \times 10^{6} \mathrm{~S} / \mathrm{m}\right)$. The difference in the values of conductivity obtained by simulation in COMSOL and using Eq. (1) is quite significant. This discrepancy can be explained by the inapplicability of the above-mentioned perturbation formula for the case of the WGM resonator with graphene. The conductivity was not calculated using Eq. (1) for the "bottom" position because of strong perturbation of the resonator by a substrate with graphene. It should be noted that we used the $\mathrm{HE}$ mode, the characteristic feature of which is the predominance of the longitudinal microwave electric field $\mathrm{E}_{\mathrm{z}}$ component over the transverse, i.e., azimuthal, field components inside the dielectric disk. Therefore, outside of the sapphire disk, at the location of graphene, a significant component of the electric field is directed perpendicular to the plane of graphene. In contrast, other works have used the TE mode resonator system, in which the electric field line is strictly parallel to the graphene plane. Therefore, the mode of polarization in the formation of the resonator response to graphene is an important issue and should be studied further.

To conclude, in this paper, the authors studied the response of a sapphire WGM resonator to a single-layer graphene film in the millimeter wave band $(\sim 40 \mathrm{GHz})$. In the resonator, the $\mathrm{HE}_{\mathrm{n} 1 \delta}$ mode with a predominant longitudinal component of the electric field inside the dielectric disk was excited. It was shown that the main features of the response of the WGM resonator with graphene are preserved in comparison with the response of the DR with the lowest $\mathrm{HE}_{01 \delta}$ mode, where the electric field line is strictly parallel to the graphene plane.

At the same time, comparing the experimental and simulation data for the resonator response to single-layer graphene transferred onto a substrate of quartz and the response without graphene allowed us to find the conductivity for graphene of known thickness and/or the graphene sheet resistance. A comparison of the graphene conductivity values obtained using COMSOL and the expression obtained by the perturbation method revealed a significant difference in the conductivity values. This difference can be explained by the validity violation of the approximations described by Eq. (1) for the case of a WGM resonator with graphene. Hence, it is important to further study the microwave response of the WGM resonator with the graphene film as a function of mode polarization as well as different graphene quality and substrate materials must be further explored. Nonetheless, at the moment, it can be claimed that the resonator technique can be used to reveal the conductivity/sheet resistance of graphene in the millimeter wave band in a contactless and noninvasive way. Moreover, the demonstrated WGM resonator technique paves the way for contactless studies of one-atomthick materials in a wide range of wavelengths including sub-mm waves.

${ }^{1}$ K. S. Novoselov, V. I. Fal'ko, L. Colombo, P. R. Gellert, M. G. Schwab, and K. Kim, Nature 490, 192-200 (2012).

${ }^{2}$ D. Kireev, I. Zadorozhnyi, T. Qiu, D. Sarik, F. Brings, T. Wu, S. Seyock, V. Maybeck, M. Lottner, B. M. Blaschke, J. Garrido, X. Xie, S. Vitusevich, B. Wolfrum, and A. Offenhaeusser, IEEE Trans. Nanotechnol. 16, 140-147 (2017).

${ }^{3}$ A. V. Emelianov, D. Kireev, D. D. Levin, and I. I. Bobrinetskiy, Appl. Phys. Lett. 109, 173101 (2016).

${ }^{4}$ J. Krupka, Meas. Sci. Technol. 19, 065701 (2008).

${ }^{5}$ J. Krupka and W. Strupinski, Appl. Phys. Lett. 96, 082101 (2010).

${ }^{6}$ J. Krupka, W. Strupinski, and N. Kwietniewski, J. Nanosci. Nanotechnol. 11, 3358-3362 (2011).

${ }^{7}$ L. Hao, J. Gallop, S. Goniszewski, O. Shaforost, N. Klein, and R. Yakimova, Appl. Phys. Lett. 103, 123103 (2013).

${ }^{8}$ L. Hao, J. Gallop, Q. Liu, and J. Chen, IET Circuits, Devices Syst. 9, 397-402 (2015).

${ }^{9}$ O. Shaforost, K. Wang, S. Goniszewski, M. Adabi, Z. Guo, S. Hanham, J. Gallop, L. Hao, and N. Klein, J. Appl. Phys. 117, 024501 (2015).

${ }^{10}$ A. Barannik, N. Cherpak, A. Kirichenko, Y. Prokopenko, S. Vitusevich, and V. Yakovenko, Int. J. Microwave Wireless Technol. 9, 781-796 (2017).

${ }^{11}$ A. A. Barannk, N. T. Cherpak, and A. N. Stetsenko, Telecommun. Radio Eng. 65, 1783-1788 (2006).

${ }^{12}$ A. A. Barannik, N. T. Cherpak, I. A. Protsenko, and S. A. Vitusevich, "Unusual millimetre wave effects in sapphire WGM resonator with ultrathin conductor films," IEEE Microwave Wirel. Compon. Lett. (unpublished).

${ }^{13}$ See https://www.comsol.com/ for details on COMSOL Multiphysics modeling using transition boundary conditions.

${ }^{14}$ See https://www.graphenea.com/ for the information on graphene used in this work.

${ }^{15}$ X. Liang, B. A. Sperling, I. Calizo, G. Cheng, C. A. Hacker, Q. Zhang, Y. Obeng, K. Yan, H. Peng, Q. Li, X. Zhu, H. Yuan, A. R. Walker, Z. Liu, L. M. Peng, and C. A. Richter, ACS Nano 5, 9144-9153 (2011). 